\title{
Antimicrobianos en Unidades de Cuidados Intensivos: Formas de administración
}

\author{
CARLOS BELTRÁN B*
}

\section{Antimicrobial agents in Intensive Care Units: Forms of administration}

Nosocomial infections demand an exceptional antiinfective therapy because of its severity and its resistance to first line antibiotics. Intensive care units must implement policies to avoid the selection of multiresistant strains that supersede the efficacy of these antibiotics; useful to reduce this selective process are: reduction of surgical prophylaxis practice, cycling of antibiotics, shortage of treatment schedules and to implement the de-escalating therapy of antimicrobial agents based on a rapid and sensible microbiological diagnosis. Multiple strategies proposed to optimize the antiinfective effect of antibiotic treatment are reviewed: application of pharmacokinetic and pharmacodinamic concepts, use of new lipid formulations of amphotericin B, inhalatory aminoglycosides and lock therapy for central vascular catheters infections. Selective decontamination in mechanically ventilated patients and topical mupirocin treatment of methicillin resistant Staphylococcus aureus nasal carriers are tools to prevent some severe infections.

Key words: Intensive care unit; Multiresistance; Strategies; Antibiotic control.

Pese a que los antimicrobianos se han usado en la práctica clínica desde el año $1935^{1}$, al avance en la comprensión de sus mecanismos de acción y formas de utilización y al desarrollo de numerosas familias de antibacterianos, la incidencia de sepsis continúa en aumento ${ }^{2}$. Por otra parte, la tasa de mortalidad por sepsis no ha disminuido significativamente aún cuando las técnicas de soporte vital implementadas en las Unidades de Cuidados Intensivos (UCI) han experimentado notables progresos. La escasez de terapias efectivas para detener los mecanismos de la respuesta inflamatoria sistémica gatillados por las infecciones severas lleva, en un alto porcentaje de los casos, al fracaso de la terapia antimicrobiana y de soporte vital. De allí que el uso adecuado y oportuno de antimicrobianos continúa siendo el pilar fundamental del manejo de las infecciones graves en UCI.

\section{Estrategias de uso de antimicrobianos en Unidades de Cuidados Intensivos}

En las UCI se concentran pacientes con disrupción de sus barreras defensivas mecánicas por el uso de catéteres, tubos traqueales, etc. Además, se seleccionan agentes bacterianos más resistentes y se dan circunstancias que facilitan la transmisión entre pacientes, por manoportación o vectores, y conducen a colonización e infecciones de difícil tratamiento.

La mortalidad de la sepsis aumenta de 56 a $78 \%$ cuando el antimicrobiano administrado inicialmente no es apropiado ${ }^{3}$ lo que justifica el inicio de tratamientos antimicrobianos empíricos de amplio espectro. En la neumonía asociada a ventilación mecánica también tiene alto impacto en la sobreviva la administración oportuna de antimicrobianos apropiados. Por otra parte, en nuestro país la tasa de resistencia a antimicrobianos es alta entre agentes aislados en procesos invasores nosocomiales ${ }^{4}$ con lo que el tratamiento empírico generalmente incluye vancomicina y carbapenems o cefalosporinas de amplio espectro. Esto, a su vez, constituye una fuerte presión para la selección de cepas resistentes y perpetúa las altas tasas de resistencia a antimicrobianos en UCI.

Se han diseñado estrategias para reducir esta resistencia en UCI que requieren políticas estrictas de uso de antimicrobianos ${ }^{5}$ tales como la reducción de la duración de la profilaxis antibacteriana quirúrgica y en el trauma ${ }^{6}$ y la rotación periódica de antimicrobianos empíricos ${ }^{7,8}$. Hay estudios que muestran que la duración del tratamiento antimicrobiano es uno de los factores

\footnotetext{
* Unidad de Infectología, Servicio de Medicina, Hospital Barros Luco-Trudeau

E-mail: cabel@vtr.net
} 
más importantes para el desarrollo de resisten$\operatorname{cia}^{9,10}$. En consideración a la necesidad de una cobertura antibacteriana precoz y adecuada, y al impacto en el panorama de resistencia de la prolongación del uso de antimicrobianos de amplio espectro, se ha desarrollado la estrategia llamada desintensificación o de - escalamiento: en los pacientes con infecciones graves se toman las muestras bacteriológicas apropiadas y se inicia un esquema antimicrobiano empírico de amplio espectro; una vez identificado el agente se reduce el espectro de la terapia mediante la suspensión de los antimicrobianos innecesarios y eventualmente la modificación del antibacteriano activo por otro de espectro más estrecho, si ello es posible. Esta conducta no aumenta la mortalidad ni la duración de la estadía en UCI y, por el contrario, reduce significativamente la incidencia de resistencia y sobreinfección ${ }^{11}$. El avance tecnológico que representan los hemocultivos automatizados en la sensibilidad y el tiempo de latencia, junto con la estandarización de las técnicas de obtención de muestra de cepillado bronquial, lavado broncoalveolar y aspirados traqueales que permiten estudios cuantitativos, han sido decisivos para poder implementar la estrategia de escalamiento.

Otro aspecto fundamental en el uso de antimicrobianos en UCI es la utilización de dosis e intervalos de administración adecuados, que permitan la máxima supresión del inóculo bacteriano reduciendo la ventana de selección de mutaciones. Para ello es necesario conocer la farmacocinética de los antimicrobianos utiliza$\operatorname{dos}^{12}$.

\section{Consideraciones farmacocinéticas e interva- los de administración}

Los primeros tratamientos con penicilina datan de 1941 y ya en 1946 Jawetz asoció el éxito del tratamiento con la presencia de niveles detectables del antibacteriano. La administración de un fármaco genera una curva de concentración que alcanza una concentración máxima (pico) y una duración en el tiempo de concentraciones detectables. Ambas variables determinan un área bajo la curva (AUC) (Figura 1). Las bacterias sensibles se caracterizan por inhibir su crecimiento a una determinada concentración del antimicrobiano (concentración inhibitoria mínima -CIM) y son destruidas por una concentración del antibimicrobiano denominada concentración bactericida mínima (CBM). Las bacterias resistentes no inhiben su crecimiento frente al antimicrobiano o exhiben CIM mayores a las

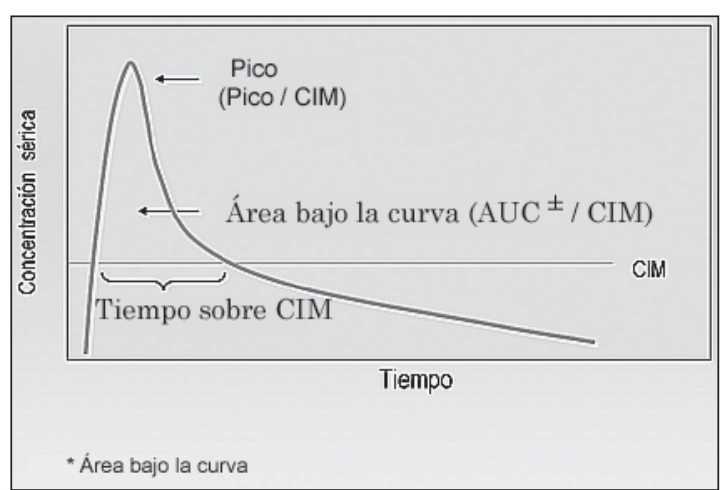

Figura 1. Concentración sérica versus tiempo en relación a CIM.

concentraciones que el antibacteriano puede alcanzar en el organismo. Las bacterias tolerantes exhiben CIM alcanzables pero tiene una fuerte disociación CIM/CBM por lo que el antimicrobiano sólo ejerce acción bacteriostática pero no bactericida $^{13}$.

El perfil concentración - tiempo es responsable de la potencia y toxicidad del antimicrobiano. Para que ejerza una adecuada acción antibacteriana, el fármaco debe alcanzar concentraciones plasmáticas en general 4 veces superiores a la CIM, para superar la CBM y en consideración a que las concentraciones tisulares son menores a las plasmáticas. En el paciente crítico los aspectos farmacocinéticos adquieren aún mayor relevancia por cuanto se ha descrito un mayor volumen de distribución de los fármacos que determina niveles plasmáticos más bajos. Por otra parte, con frecuencia las infecciones son causadas por agentes más resistentes, vale decir con CIM más altas, por lo que la obtención de concentraciones adecuadas tanto en el plasma como en el sitio de infección es fundamental para el éxito de la terapia pero también para prevenir la aparición de más resistencia al antimicrobiano. Bernard describe una concentración del antibacteriano denominada concentración de prevención de mutaciones $(\mathrm{CPM})^{14}$. Las concentraciones del antimicrobiano entre la CIM y la CPM

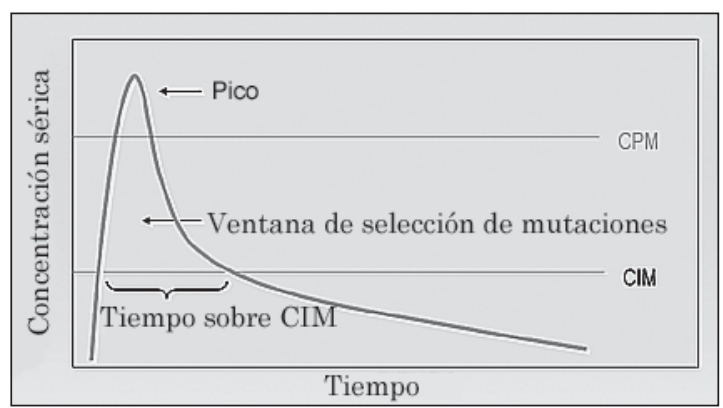

Figura 2. Concentración sérica versus CIM Y CPM 


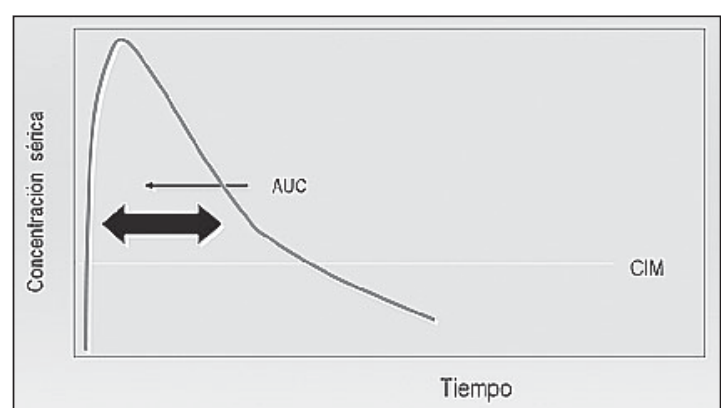

Figura 3. Muerte bacteriana dependiente de tiempo ( $\beta$ lactámicos, glicopéptidos)

constituyen la ventana de selección de mutaciones de resistencia y aumentan el pool genético de resistencia de la Unidad (Figura 2). También se debe tener en cuenta que, con frecuencia, los pacientes hospitalizados en las UCI tienen disfunción de diversa magnitud de los órganos excretores, especialmente insuficiencia renal, lo que contribuye a la variabilidad interindividual de los niveles plasmáticos de los fármacos, requiriendo ajustes de dosis y medición de niveles para antimicrobianos con margen terapéutico estrecho. Cada antimicrobiano tiene un mecanismo de acción específico que determina que algunos posean acción bactericida constante sobre la CBM y otros se caractericen por una acción bactericida variable dependiente de la concentración, es decir a mayor concentración la velocidad bactericida es mayor.

Los $\beta$ lactámicos tienen acción bactericida constante independientemente de que la concentración esté en el pico o apenas sobre la CIM por lo que el objetivo del tratamiento es el lograr que el nivel plasmático del antibacteriano se encuentre sobre la CIM el mayor tiempo posible (Figura $3)$. Estos antimicrobianos requieren ser administrados en intervalos frecuentes dependiendo de la vida media específica del fármaco utilizado para obtener niveles sobre la CIM durante al menos 30 a $40 \%$ del intervalo entre dosis. Se ha sugerido que en pacientes críticos deberían lograrse niveles sobre la CIM por más del $50 \%$ del intervalo entre dosis lo que ha llevado a su uso en infusión continua, especialmente en el caso de las cefalosporinas que, por su acción sobre las PBP3, tienen una acción bactericida más lenta. Los estudios realizados con cefalosporinas utilizando dosis de carga e infusión continua demuestran mejor perfil farmacocinético con tiempos más prolongados sobre la CIM y leve menor penetración a tejidos; sin embargo, ello no se ha traducido en mayores tasas de curación ni en reducción de los días de estada en $\mathrm{UCI}^{15,16}$. El alto índice de seguridad de los $\beta$ lactámicos que permite altas

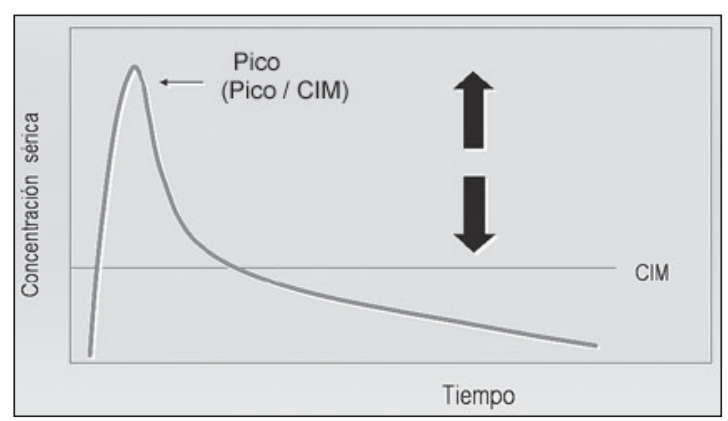

Figura 4. Muerte bacteriana dependiente de concentración (aminoglucósidos, fluoroquinolonas)

dosificaciones avala su eficacia independientemente de la forma de administración y no hay pruebas definitivas de superioridad con el uso en infusión respecto de la administración intermitente. Sin embargo, en infecciones graves y frente a ciertos agentes como Pseudomonas aeruginosa o cocáceas Gram positivas debe considerarse acortar los intervalos de administración habituales o incluso la administración continua con dosis de carga inicial y mediciones de niveles plasmáticos en el estado estacionario, que se produce después de las 5 horas de infusión.

Anfotericina B es otro antimicrobiano que se ha utilizado en infusión continua con el objeto de reducir la toxicidad, en particular la relativa a la velocidad de infusión mediada por liberación de citoquinas, y permitir dosificaciones diarias mayores para micosis parcialmente resistentes. Un estudio reciente en pacientes neutropénicos demostró que esta modalidad de administración permite alcanzar dosis diarias de hasta 2,0 mg/kg/día en escalamiento progresivo, con menor incidencia de reacciones adversas y similar eficacia ${ }^{17}$.

A diferencia de los $\beta$ lactámicos, los aminoglucósidos y las quinolonas se caracterizan por una curva bactericida concentración dependiente obteniéndose la mayor velocidad bactericida con concentraciones cercanas al pico. Además estos fármacos tienen un efecto post antibiótico intenso contra diferentes especies bacterianas, vale decir que la acción inhibitoria continúa después que el antimicrobiano ha sido removido del entorno bacteriano. El objetivo es, por tanto, alcanzar picos muy altos mediante la administración de dosis elevadas en forma espaciada, con lo que se obtiene la mayor eficacia y menor riesgo de aparición de resistencia (Figura 4). Los aminoglucósidos son antibacterianos antiguos pero que siguen siendo de gran utilidad en UCI. Poseen un estrecho margen terapéutico por lo que su uso prolongado requiere medición de niveles pico y valle. Distintos estudios realizados en pacientes no críticos confirman la eficacia de los 
aminoglucósidos utilizados en dosis total única diaria y, debido a que la toxicidad está relacionada con niveles valle elevados que se evitan con esta modalidad de administración, se ha observado menor toxicidad. Buijk estudió la farmacocinética de gentamicina y tobramicina en dosis unitaria diaria en pacientes críticos encontrando gran variabilidad interindividual en los niveles plasmáticos, lográndose en la mayoría de los casos concentraciones máximas 10 veces superiores a la CIM $^{18}$. Estos hallazgos confirman el potencial uso de aminoglucósidos en dosis unitaria diaria en UCI y la necesidad de controlar niveles plasmáticos en pacientes críticos.

\section{Anfotericina B en infusiones lipídicas}

En la década de los 90 se desarrollaron comercialmente distintas formulaciones lipídicas de anfotericina cuya menor toxicidad permite la administración de dosis diarias superiores incluso a $\operatorname{los} 5,0 \mathrm{mg} / \mathrm{kg} / \mathrm{dí}$. De los tres tipos de presentaciones lipídicas: ABLC (anfotericina en complejos lipídicos), ABCD (anfotericina de dispersión coloidal) y anfotericina liposomal (Ambisome ${ }^{\mathrm{R}}$ ), esta última es la más utilizada si bien en Chile su penetración ha sido baja por los altos costos que involucra esta terapia y por el advenimiento de nuevas y promisorias moléculas antifúngicas.

Diversos estudios muestran disminución de la toxicidad renal y de la toxicidad mediada por citoquinas; sin embargo, no se ha logrado un aumento significativo en la eficacia de anfotericina en infecciones micóticas filamentosas invasoras. La eficacia global en distintos estudios es variable entre 40 y $60 \%$. Incluso estudios que comparan distintas dosificaciones de una misma anfotericina lipídica han fallado en demostrar mayor eficacia con dosis más altas que con dosis intermedias ${ }^{19,20}$. En la actualidad las formulaciones lipídicas se utilizan principalmente para continuar tratamiento en presencia de fracaso o toxicidad por anfotericina convencional.

\section{Usos tópicos de antimicrobianos en UCI}

Desde hace largos años se vienen ensayando aplicaciones tópicas de ciertos antimicrobianos para la prevención de infecciones $y$, en algunos casos, para el tratamiento de ellas una vez producidas. En esta ocasión nos referiremos a la descontaminación nasal y del tubo digestivo, al uso de antimicrobianos por vía inhalatoria y a la técnica de sellado con soluciones de antibacterianos (antibiotic - lock) en catéteres centrales.
Entre 25 y $30 \%$ de la población general desarrolla portación nasal de Staphylococcus aureus. En algunos contextos especiales como pacientes hospitalizados, diabéticos, drogadictos o en diálisis se puede comprobar portación nasal por $S$. aureus resistente a meticilina (cloxacilina) (SAMR). En más del $80 \%$ de los casos, cuando estos pacientes desarrollan bacteriemia, la cepa aislada en sangre es idéntica a la encontrada en fosas nasales. Un estudio reciente confirma este hallazgo y demuestra que la portación nasal por SAMR es un factor de riesgo para bacteriemia por SAMR ${ }^{21}$. Se ha utilizado mupirocina endonasal para erradicar $S$. aureus con el objeto de reducir la incidencia de bacteriemia estafilocóccica en pacientes hospitalizados. Perl utilizó mupirocina tópica versus placebo cada 12 horas en 3.864 pacientes preoperatorios sin lograr una reducción significativa de la incidencia de bacteriemia estafilocóccica en el grupo con mupirocina ${ }^{22}$; $\sin$ embargo, mupirocina mostró un alta tasa de erradicación en portadores y, al circunscribir el análisis de eficacia al grupo de pacientes previamente portadores de $S$. aureus, se observó una reducción de la tasa de bacteriemia de 7,7 a 4,0\%. En resumen la evidencia actual no apoya el uso generalizado de mupirocina pero, en pacientes portadores, su uso reduce la incidencia de bacteriemia.

La descontaminación selectiva del tubo digestivo se basa en el reconocimiento de este tracto como reservorio bacteriano y micótico a partir del cual se genera una parte importante de las infecciones nosocomiales por agentes resistentes que rápidamente colonizan a los pacientes hospitalizados, reemplazando a la flora nativa. Consiste en la administración oral o por sonda de combinaciones de antimicrobianos no absorbibles de amplio espectro, habitualmente polimixina más tobramicina y anfotericina, junto con un antimicrobiano endovenoso de amplio espectro como cefotaxima por los primeros 4 días. Esta técnica se viene utilizando desde los años 80 principalmente en Europa y no ha tenido gran aceptación en E.U.A. ni en nuestro medio por sus altos costos, la falta de disponibilidad de soluciones para uso tópico y por diversos estudios que han demostrado su asociación con el incremento de resistencia. Existen al menos 25 estudios y 4 meta-análisis que demuestran, sin embargo, su eficacia en reducir la incidencia de neumonía, bacteriemia e infección urinaria en pacientes quirúrgicos con una reducción de $30 \%$ en mortalidad. En pacientes críticos no quirúrgicos la eficacia ha sido menor y no se ha logrado el mismo 
impacto en mortalidad ni en la reducción de los días de estada en $\mathrm{UCI}^{23}$. No existe consenso para su uso sistemático e incluso algunos autores desaconsejan su uso por el aumento en la incidencia de cepas resistentes ${ }^{5}$.

Los antimicrobianos inhalatorios han sido utilizados como tratamiento de infecciones respiratorias asociadas a intubación traqueal y en un intento de prevenir la neumonía asociada a ventilación mecánica, pero no han demostrado eficacia en este contexto y han sido vinculados a aumento de la resistencia bacteriana, por lo que no hay fundamentos para su uso clínico pese a que hay estudios que demuestran que disminuyen la adherencia bacteriana al tubo traqueal y la formación de biofilm ${ }^{24}$. Sin embargo, los antimicrobianos inhalatorios han demostrado utilidad en otros contextos como pentamidina en la prevención de la neumonía por Pneumocystis jiroveci (ex carinii) en VIH/ SIDA y aminoglucósidos en prevención de las infecciones por Pseudomonas spp, contribuyendo a la mejoría de la función respiratoria en niños con fibrosis quística. También se ha demostrado la eficacia de anfotericina en nebulizaciones para la prevención de las infecciones pulmonares por Aspergillus spp en pacientes con transplante cardíaco o pulmonar con una reducción significativa de los eventos infecciosos ${ }^{25}$.

Pero quizás si una de las formas más novedosas de uso de antimicrobianos tópicos la constituye el sellado interno de catéteres centrales con soluciones de antibióticos. Esta técnica conocida como antibiotic - lock (o terapia de candado) fue usada inicialmente en pacientes pediátricos con catéteres implantados y consiste en rellenar, después de su uso, el espacio muerto del catéter con aproximadamente $2 \mathrm{ml}$ de una solución de anfotericina, vancomicina o aminoglucósidos, pero esta práctica se ha extendido a otros tipos de catéteres. En pacientes neutropénicos se ha demostrado una reducción significativa de las bacteriemias ${ }^{26}$ y en catéteres de hemodiálisis se ha demostrado disminución significativa de la tasa de infección local y de bacteriemia con un aumento neto en la vida útil de los catéteres; sin embargo, se han reportado casos con niveles plasmáticos detectables y aún en rango tóxico del antimicrobiano usado localmente ${ }^{27}$. También se han reportado casos anecdóticos de tratamiento de infecciones de catéter mediante esta técnica, exclusiva o asociada a antimicrobianos sistémicos, sin recurrir a su remoción. El impacto de esta técnica en la epidemiología de la resistencia a antimicrobianos aún no ha sido evaluado.

\section{Conclusiones}

El uso de antimcrobianos en las UCI sigue constituyendo una herramienta fundamental para el tratamiento de las infecciones graves. La prescripción precoz de un tratamiento antimicrobiano apropiado tiene gran impacto en la sobreviva de la sepsis y la neumonía asociada a ventilación mecánica. Por esta razón el tratamiento antimicrobiano empírico constituye una estrategia de eficacia probada en infecciones graves en UCI y debe basarse en el panorama epidemiológico local de resistencia. Las estrategias de de-escalamiento y rotación de antimicrobianos empíricos, como también la racionalización en la duración de los tratamientos y de la profilaxis antibacteriana, han demostrado reducir el impacto negativo del uso empírico de antimicrobianos de amplio espectro.

El conocimiento de la farmacocinética y farmacodinamia de los antimicrobianos es fundamental para la prescripción de dosis e intervalos adecuados de los antimicrobianos elegidos. Los esquemas de dosis única diaria en antibacterianos con actividad concentración dependiente, como aminoglucósidos, han permitido reducir la toxicidad de esta familia de antimicrobianos conservando, al menos, la misma eficacia. El amplio margen de seguridad de los $\beta$ lactámicos ha permitido su éxito aún cuando son utilizados en dosis intermitentes. Hay estudios clínicos que avalan las ventajas farmacocinéticas del uso de cefalosporinas en infusión continua sin que, a la fecha, se haya demostrado beneficio clínico de esta forma de administración.

Las formulaciones lipídicas de anfotericina han permitido disminuir su toxicidad y administrar dosis diarias mayores; sin embargo, no constituyen la primera elección en micosis invasoras y su uso se ha restringido más bien a pacientes que han experimentado toxicidad con anfotericina deoxicolato (convencional) o a situaciones de rescate por fracaso de la terapia estándar.

A pesar de los beneficios que ha demostrado la descontaminación selectiva del tubo digestivo, no se aconseja su uso rutinario por sus altos costos y el impacto sobre la resistencia. En general no se recomienda el uso de antimicrobianos tópicos en pacientes de UCI, pudiendo considerarse como excepción la mupirocina nasal en la erradicación de portadores de $S$. aureus resistente a meticilina que ingresan a UCI, las nebulizaciones de anfotericina en transplante de corazón y pulmón o el sellado de catéteres de 
larga permanencia con antimicrobianos, especialmente en pacientes con accesos vasculares difíciles.

\section{Resumen}

Las infecciones nosocomiales en pacientes críticos exigen de una terapia antinfecciosa de excepción por la gravedad de las mismas y por su refractariedad a los antimicrobianos habituales. En las unidades de pacientes críticos deben implementarse políticas para evitar la selección de cepas multiresistentes que comprometa la eficacia de estos antimicrobianos; útiles para limitar esta selección son: reducción en el uso de profilaxis quirúrgica, rotación de antimicrobianos, abreviar los plazos de tratamiento e implementar el de-escalamiento de agentes antibacterianos apoyado en un diagnóstico microbiológico rápido y sensible. Se revisan las múltiples estrategias propuestas para optimizar el efecto antimicrobiano de los antibióticos: aplicación de conceptos farmacocinéticos y farmacodinámicos, uso de nuevas presentaciones lipídicas de anfotericina B, el empleo inhalatorio de aminoglucósidos y la terapia «de candado» para las infecciones de catéteres vasculares centrales. Se discuten también la descontaminación selectiva en el paciente en ventilación mecánica y el tratamiento con mupirocina tópica de la portación nasal de Staphylococcus aureus resistente a meticilina como herramientas para prevenir algunas infecciones severas.

\section{Bibliografía}

1.- Carithers H. The first use of an antibiotic in America. Am J Dis Child 1974; 128: 207-11.

2.- Martin G, Mannino D, Eaton S, Moss M. The epidemiology of sepsis in the United States from 1979 through 2000. N Engl J Med 2003; 348: 1546-54.

3.- Leone $\mathrm{M}$, Bourgoin A, Cambon $\mathrm{S}$ et al. Empirical antimicrobial therapy of septic shock patients: adequacy and impact on the outcome. Crit Care Med 2003; 31: 648-50.

4.- Trucco O, Prado V, Durán C y grupo Pronares. Red de vigilancia de resistencia antimicrobiana Pronares. Rev Chil Infect 2002; 19(S2): S140-8.

5.- Kollef M, Fraser V. Antibiotic resistance in the Intensive Care Unit. Ann Intern Med 2001; 134: 298-314.

6.- Velmahos G, Toutouzas K, Sarkisyan G et al. Severe trauma is not an excuse for prolonged antibiotic prophylaxis. Arch Surg 2002; 167: 537-41.

7.- Gruson D, Hibert G, Vargas F et al. Rotation and restricted use of antibiotics in a Medical Intensive Care Unit. Impact on the incidence of ventilator associated pneumonia caused by antibiotic resistant gram - negative bacteria. Am J Respir Crit Care Med 2000; 162: 837-43.

8.- Moss W, Beers M, Johnson E et al. Pilot study of antibiotic cycling in a pediatric ICU. Crit Care Med
2002; 30: 1877-82.

9.- Namias N, Harvill S, Ball S et al. Cost and morbidity associated with antibiotic prophylaxis in the ICU. J Am Coll Surg 1999; 188: 225-30.

10.- Namias N, Harvill S, Ball S et al. Empiric therapy of sepsis en the Surgical Intensive Care Unit with broad - spectrum antibiotics for 72 hours does not lead to the emergence of resistant bacteria. J Trauma 1998; 45: 887-91.

11.- Singh N, Rogers P, Atwood C et al. Short - course empiric antibiotic therapy for patients with pulmonary infiltrates in the Intensive Care Unit. A proposed solution for indiscriminate antibiotic prescription. Am J Respir Crit Care Med 2000; 162: 505-11.

12.- Álvarez, Lerma F, Palomar M, Grau S. Management of antimicrobial use in the Intensive Care Unit. Drugs 2001; 61: 763-75.

13.- Amsden G W, Ballow C H, Bertino J S Jr. Pharmacokinetics and Pharmacodynamics of AntiInfective Agents. En Mandell, Douglas and Bennett's Principles and Practice of Infectious Diseases. Mandell Gl, Bennett JE and Dolin R, editors. 5th edition 2000. Churchill Livingstone, Philadelphia, pp 253-60.

14.- Bernard E, Breilh D, Bru J et al. Is there a rationale for continuous infusion of cefepime? A multidisciplinary approach. Clin Microbiol Infect 2003; 9: 339-48.

15.- Nicolau D, McNabb J, Lacy M et al. Pharmacokinetics and pharmacodynamics of continuous and intermittent ceftazidime during the treatment of nosocomial pneumonia. Clin Drug Invest 1999; 18: 133-9.

16.- Georges B, Archambaud M, Saivin S et al. Perfusion continue versus administration discontinue de céfépime en réanimation. Résultats préliminaires. Path Biol 1999; 47: 483-5.

17.- Imhof A, Wlater R, Schaffner A. Continuous infusion of escalated doses of amphotericin B deoxycholate: an open - label observational study. Clin Infect Dis 2003; 36: 943-51.

18.- Buijk S, Mouton J, Gyssens I et al. Experience with a once - daily dosing program of aminoglycosides in critically ill patients. Intensive Care Med 2002; 28: 936-42.

19.- Ellis M, Spence B, De Paw B et al. An EORTC international multicenter trial comparing two dosages of liposomal amphotericin B for treatment of invasive aspergillosis. Clin Infect Dis 1998; 27: 1406-12.

20.- Walsh T, Hiemenz J, Seibel N et al. Amphotericin B lipid complex for invasive fungal infections: analysis of safety and efficacy in 556 cases. Clin Infect Dis 1998; 26: 1383-96.

21.- Von Eiff C, Becker K, Machka K et al. Nasal carriage as a source of Staphylococcus aureus bacteremia. N Engl J Med 2001; 344: 11-6.

22.- Perl T, Cullen J, Wenzel R et al. Intranasal mupirocin to prevent postoperative Staphylococcus aureus infections. N Engl J Med 2002; 346: 1871-77.

23.- Nathens A, Marshall J. Selective decontamination of the digestive tract in surgical patients. A systematic review of the evidence. Arch Surg 1999; 134: 170-76.

24.- Adair C, Gorman S, Byers L et al. Eradication of endotracheal tube biofilm by nebulised gentamicin. Intensive Care Med 2002; 28: 426-31.

25.- Reichenspurner H, Gamberg P, Nitschke $M$ et al. Significant reduction in the number of fungal infections after lung, heart - lung and heart transplantation using aerosolized amphotericin B prophylaxis. Transplant Proc 1997; 29: 627-8.

26.- Carratalá J, Niubo J, Fernández - Sevilla A et al. . 
Randomized, double - blind trial of an antibiotic lock technique for prevention of gram - positive central venous catheter - related infection in neutropenic patients with cancer. Antimicrob Agents Chemother 1999; 43: 2200-4.
27.- Dogra G, Herson H, Hutchinson B et al. Prevention of tunneled hemodyalisis catheter - related infections using catheter - restricted filling with gentamicin and citrate: a randomized controlled study. J Am Soc Nephrol 2002; 13: 2133-9. 